\title{
Antifungal Activity of Novel Formulations Based on Terpenoid Prodrugs against $C$. albicans in a Mouse Model
}

\author{
Suvidha Menon ${ }^{1}$, Xiuyi Liang ${ }^{1}$, Richa Vartak ${ }^{1}$, Ketankumar Patel ${ }^{1}$, Antonio Di Stefano ${ }^{2}{ }^{(D}$, Ivana Cacciatore ${ }^{2}(\mathbb{D}$, \\ Lisa Marinelli ${ }^{2, *}$ and Blase Billack ${ }^{1, *} \mathbb{E}$
}

check for

updates

Citation: Menon, S.; Liang, X.;

Vartak, R.; Patel, K.; Di Stefano, A.;

Cacciatore, I.; Marinelli, L.; Billack, B.

Antifungal Activity of Novel

Formulations Based on Terpenoid

Prodrugs against $C$. albicans in a

Mouse Model. Pharmaceutics 2021, 13,

633. https://doi.org/10.3390/

pharmaceutics 13050633

Academic Editor: Gert Fricker

Received: 11 March 2021

Accepted: 23 April 2021

Published: 29 April 2021

Publisher's Note: MDPI stays neutral with regard to jurisdictional claims in published maps and institutional affiliations.

Copyright: (C) 2021 by the authors Licensee MDPI, Basel, Switzerland. This article is an open access article distributed under the terms and conditions of the Creative Commons Attribution (CC BY) license (https:// creativecommons.org/licenses/by/ $4.0 /)$.
1 Department of Pharmaceutical Sciences, College of Pharmacy and Health Sciences, St. John's University, 8000 Utopia Parkway, Jamaica, NY 11439, USA; menons@stjohns.edu (S.M.); liangx@stjohns.edu (X.L.); vartakr@stjohns.edu (R.V.); patelk@stjohns.edu (K.P.)

2 Department of Pharmacy, “G. D'Annunzio” University of Chieti-Pescara, 66100 Chieti Scalo, Italy; antonio.distefano@unich.it (A.D.S.); ivana.cacciatore@unich.it (I.C.)

* Correspondence: 1.marinelli@unich.it (L.M.); billackb@stjohns.edu (B.B.); Tel.: +39-871-355-4475 (L.M.); +1-718-990-5657 (B.B.)

Abstract: Carvacrol (CAR), a phenolic monoterpenoid, has been extensively investigated for its antimicrobial and antifungal activity. As a result of its poor physicochemical properties, water soluble carvacrol prodrugs (WSCPs) with improved water solubility were previously synthesized and found to possess antimicrobial activity. Here, three novel CAR analogs, WSCP1, WSCP2, and WSCP3, were tested against fluconazole (FLU)-sensitive and -resistant strains where they showed greater antifungal activity than CAR against $C$. albicans. The probable mechanism by which the CAR prodrugs exert the antifungal activity was studied. Results from medium acidification assays demonstrated that the CAR and its synthetically designed prodrugs inhibit the yeast plasma membrane H+-ATPase (Pma1p), an essential target in fungi. In other words, in vitro data indicated that CAR analogs can prove to be a better alternative to CAR considering their improved water solubility. In addition, CAR and WSCP1 were developed into intravaginal formulations and administered at test doses of $50 \mathrm{mg} / \mathrm{kg}$ in a mouse model of vulvovaginal candidiasis (VVC). Whereas the CAR and WSCP1 formulations both exhibited antifungal efficacy in the mouse model of VVC, the WSCP1 formulation was superior to CAR, showing a remarkable decrease in infection by $\sim 120$-fold compared to the control (infected, untreated animals). Taken together, a synthetically designed prodrug of CAR, namely WSCP1, proved to be a possible solution for poorly water-soluble drugs, an inhibitor of an essential yeast pump in vitro and an effective and promising antifungal agent in vivo.

Keywords: antifungal; Candida albicans; carvacrol; intravaginal formulations; minimum inhibitory concentration

\section{Introduction}

Opportunistic fungal infections primarily caused by Candida albicans (C. albicans) are common. Despite being a commensal colonizer in various human niches (the oral mucosa, the gut, the vaginal tract, and the skin), C. albicans can successfully evolve as a pathogen because of its adaptability to the changing host microbiome (such as antibiotic treatment or when the host becomes debilitated or immunocompromised). The standard antifungal drug, fluconazole (FLU), is fungistatic in nature, and hence, is a concern to immunocompromised patients. The high mortality rates associated with systemic infection or blood-borne candidiasis is another growing concern. Altogether, the various evolved mechanisms to evade human immunity and the metabolic flexibility of C. albicans, the pharmacological limitations of antifungal drugs, and the growing emergence of FLUresistant as well as multidrug-resistant strains in immunocompromised patients justifies the relevance of investigation of novel antifungal targets [1,2]. 
Essential oils are volatile, complex natural mixtures of either terpenes, terpenoids, aromatic, or aliphatic compounds formed by aromatic plants. Although the use of essential oils dates to ancient times, it is only recently that they have gained great attention because of their numerous effects in various fields like the pharmaceutical, food, sanitary, cosmetic, and perfume industries. Notably, these industries are adopting the use of green products to inhibit several pathogens as well as to render the product as fit to use. Extensive research has been conducted to understand the properties of essential oils, the structure-activity relationship (SAR) of constituents of essential oils, the mechanism of action, desirable and undesirable effects, etc. Various research studies have demonstrated that essential oils possess antibacterial, antifungal, and insecticidal properties [3-6]. Several in vitro studies have shown that phenolic compounds exhibit better antimicrobial activity compared to other components of essential oils $[7,8]$.

Carvacrol (CAR, 2-methyl-5-(1-methylethyl)phenol), a phenolic monoterpenoid, is a major phytoconstituent of numerous aromatic herbs. Several insights into SAR studies have shown a link between physicochemical properties, such as hydrophobicity, the presence of a hydroxyl group, and a delocalized electron system, and carvacrol's antimicrobial activity [7,9-12]. Research data has shown that the antifungal activity of CAR can be attributed to the inhibition of ergosterol biosynthesis and the disruption of membrane integrity, which is fungicidal. The commonly used drug FLU is fungistatic, although it acts by a similar mechanism to CAR [13]. Although CAR has been extensively studied, its poor physicochemical properties hinder its use therapeutically. Numerous water-soluble CAR prodrugs (WSCPs) and codrugs have been recently synthesized $[9,14]$ to show better water solubility, thereby alleviating the bioavailability issues. In particular, in a previous study, following the prodrug approach, twenty-three CAR derivatives were prepared, and their physico-chemical and antimicrobial properties were evaluated. Among prodrugs, WSCP1-3 revealed the highest water solubility as well as antibacterial and antifungal properties against gram-negative bacteria and different species of Candida, respectively [14]. In the present study, both CAR and these three water soluble prodrugs (Figure 1) were investigated for activity against the yeast plasma membrane H+-ATPase (Pma1p), an essential pump in yeast that has been proposed to be a viable antifungal candidate drug target [15]. This was done using FLU-sensitive (S1) and FLU-resistant (S2) strains of C. albicans.

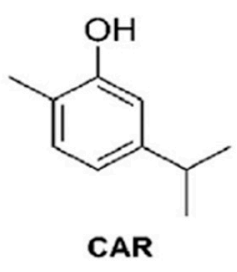
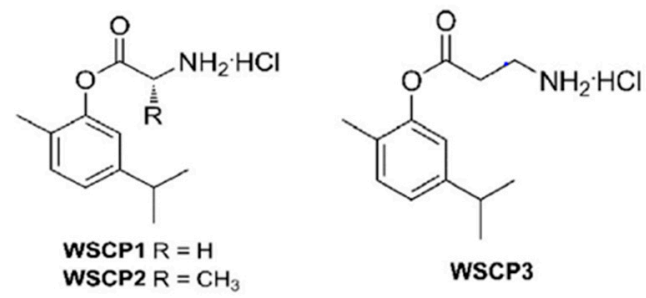

Figure 1. Chemical structures of CAR and its prodrugs.

Vulvovaginal candidiasis (VVC) is the most common infection occurring in women of childbearing age, which is caused by C. albicans [2]. A mouse model has been utilized to study vaginal yeast infections $[16,17]$. In the present study, our lab has repurposed CAR and its prodrug WSCP1 with an aim to maximize its intravaginal efficacy by developing locally delivered formulations of CAR and WSCP1 for a compatible in vivo delivery while retaining antifungal activity against the Candida strain used for VVC infection. Altogether, this is the first report that discusses novel formulations of CAR and WSCP1 as a possible intervention for VVC.

\section{Materials and Methods}

\subsection{Chemicals, Cells, and Reagents}

Dimethyl sulfoxide (DMSO) (cat\# D1435) was obtained from the Sigma Aldrich Chemicals Company (St. Louis, MO, USA). The strain of C. albicans used in this study are sensitive 
to FLU (clinical isolate S1) and resistant to FLU (clinical isolate S2) and were kindly provided by Dr. J. Morschhäuser (University of Würzburg, Würzburg, Germany) [18]. CAR and its prodrugs WSCP1-WSCP3 were synthesized as previously described $[9,14]$. FLU (cat \# B2094) was purchased from ApexBio (Boston, MA, USA). Captex ${ }^{\circledR} 300$ EP/NF (Glyceryl Tricaprylate/Tricaprate) was kindly gifted by Abitec Corp (Columbus, OH, USA). Kolliphor ${ }^{\circledR}$ HS15 (PEG-15-Hydroxystrearate) was obtained as a gift sample from BASF (New York, NY, USA). A liquid yeast-extract, peptone and dextrose (YPD) medium (pH 6.5) was prepared by adding YPD media broth powder $(50.0 \mathrm{~g})$ from HiMedia Laboratories (cat \# M1363; Mumbai, India) to distilled water ( 1000.0 mL) along with adenine hemisulfate salt (0.4 g) from Sigma Aldrich Chemicals Company (cat \# A9126; St. Louis, MO, USA) and then autoclaved at $121^{\circ} \mathrm{C}$ for $15 \mathrm{~min}$. YPD agar plates were prepared by the addition of $65 \mathrm{~g}$ of agar from Becton, Dickinson and Company (Franklin Lakes, NJ, USA; cat \# DF0427-17-6) to $1 \mathrm{~L}$ of YPD liquid medium prior to sterilization. The molten mixture was then poured into petri dishes ( $20 \mathrm{~mL} /$ dish). Roswell Park Memorial Institute (RPMI) 1640 medium, buffered with 0.165 M MOPS (3-(N-morpholino) propanesulfonic acid) containing L-glutamine and lacking sodium bicarbonate, was purchased from Sigma-Aldrich Co. (cat \# R6504; St. Louis, MO, USA). 96-well cell culture plates were purchased from Eppendorf (Hauppauge, NY, USA; Cat \# 13-690-076).

\subsection{Determination of Minimum Inhibitory Concentration (MIC) of Test Compounds (CAR and Its Prodrugs)}

The two-fold broth microdilution method with small modifications following Clinical and Laboratory Standards Institute (CLSI) document M27-A guidelines was used to determine the MIC value $[19,20]$. The final inoculum suspension contained $1-5 \times 10^{5} \mathrm{CFU} / \mathrm{mL}$ of yeast strains S1 or S2. The stock solutions of CAR, the prodrugs of CAR (WSCP1-3), and the FLU were dissolved in DMSO and diluted in RPMI 1640 medium to achieve the drug concentration (32 mM). A two-fold dilution of these test compounds was prepared in RPMI 1640 medium to obtain a concentration range of $16,8,4,2,1$, and $0.5 \mathrm{mM}$. Triplicate samples were performed for each test concentration. Each test well was filled with $200 \mu \mathrm{L}$ of the cell suspension followed by $200 \mu \mathrm{L}$ of the appropriate $2 \times$-concentration test compound. The concentration of DMSO in any well was $\leq 0.4 \%$ and did not affect yeast growth at this concentration. Two drug-free medium wells were employed to provide sterility and growth controls, whereas FLU served as a positive control. All plates were incubated at $30^{\circ} \mathrm{C}$ for $48 \pm 4 \mathrm{~h}$, and MIC values were recorded following a visual observation of turbidity as compared to the drug-free growth medium. The MIC was defined as the lowest concentration showing complete inhibition of growth after $48 \mathrm{~h}$ in all three wells of a given treatment.

\subsection{Determination of the Effect of Test Compounds (CAR and Its Prodrugs) upon Medium Acidification by S1 and S2 Strains of C. albicans}

The medium acidification assay was performed as described previously [21,22]. Considering the MIC values, the test compounds (WSCP1, WSCP2, and WSCP3 along with CAR) were used in this assay to determine the inhibitory effect, if any, at an increasing concentration $(0.1,0.3,1$, and $3 \mathrm{mM})$ on the acidification of growth medium by S1 or S2 strains. The inhibitory activity of these compounds was compared to that of FLU, which was hypothesized to possess no activity against this plasma membrane pump. The concentration of test compounds required to inhibit medium acidification by $50 \%\left(\mathrm{IC}_{50 \mathrm{MA}}\right)$ was then determined from a plot of the change in $\mathrm{pH}$ at 30 min versus the concentration of the test compound and was compared with the results for untreated cells, which were assigned the value of $100 \%$.

\subsection{Preparation of CAR and WSCP1 Formulations}

The CAR and WSCP1 formulations were prepared by using Captex ${ }^{\circledR} 300 \mathrm{EP} / \mathrm{NF}$ and Kolliphor ${ }^{\circledR}$ HS15 as solubilizing agents. For the CAR formulation, a mixture of $5 \% v / v$ carvacrol, $47.5 \%$ v $v$ Captex ${ }^{\circledR} 300 \mathrm{EP} / \mathrm{NF}$, and $47.5 \%$ v/v Kolliphor ${ }^{\circledR}$ HS15 was made. For 
the WSCP1 formulation, $5 \% w / v$ of the WSCP1 powder was thoroughly mixed in a blend of $6.25 \%$ v/v Captex $^{\circledR} 300 \mathrm{EP} / \mathrm{NF}, 6.25 \%$ v/v Kolliphor $^{\circledR}$ HS15, and $87.5 \%$ v /v purified water.

\subsection{In Vivo Antifungal Activity Assays Using CAR and WSCP1 Formulations}

Animals: female BALB/c mice 18-22 g were purchased from Taconic Laboratories, Inc. (Albany, NY, USA). Mice were maintained in an Association for Assessment and Accreditation of Laboratory Animal Care (AAALAC)-approved Animal Care Centre (ACC) at St. John's University (Jamaica, NY, USA). The study received the approval of the Institutional Animal Care and Use Committee (IACUC) of St. John's University (Protocol \# 1977). Animals were allowed to acclimatize for a week in the ACC. A previously reported mouse model of VVC was implemented here [23] with slight modifications; in particular, a clinical isolate, S1 yeast, was inoculated intravaginally.

(I) Growth conditions for Candida albicans: the $\mathrm{S} 1$ strain of $C$. albicans was grown in yeastextract-peptone-dextrose (YPD) broth for $\sim 11 \mathrm{~h}$ at $30^{\circ} \mathrm{C}$ with shaking at $200 \mathrm{rpm}$ to reach a stationary phase culture. Following incubation, the yeast culture was washed in sterile YPD and enumerated on a YPD agar plate to determine the colony forming units (CFUs $/ 100 \mu \mathrm{L}$ ).

(II) Vaginal inoculation: The murine VVC study accounted for a total of 9 days (Supplementary Materials, Figure S1). Briefly, mice were administered with $0.2 \mathrm{mg}$ of $\beta$-estradiol 17-valerate dissolved in $100 \mu \mathrm{L}$ sesame oil by subcutaneous injection $72 \mathrm{~h}$ prior to inoculation (day-3) and on day 3. Estrogenized mice were intravaginally inoculated by introducing $20 \mu \mathrm{L}$ of YPD-containing C. albicans S1 strain blastoconidia $\left(5.5 \times 10^{5} \mathrm{CFU} / 20 \mu \mathrm{L}\right)$ into the vaginal lumen (day 0$)$, and the infection was allowed to progress until day 6 . After development of the VVC model, we stepped into the intervention study. The interventions were administered intravaginally on three consecutive days (days 3, 4, and 5). All the animals were infected and then treated with the respective treatments: blank, CAR $(50 \mathrm{mg} / \mathrm{kg})$, and WSCP1 $(50 \mathrm{mg} / \mathrm{kg})$. A 6th-day-infected group served as a control for the baseline value for infection. For further information about treatment groups, see Supplementary Figure S1 in the Supplementary Materials.

(III) Endpoints: Immediately after animals were euthanized (day 6), 100 $\mu \mathrm{L}$ of vaginal lavage fluid was collected to determine the vaginal fungal burden, while the vaginal tissues were excised for histopathological analysis.

(a) Determination of vaginal fungal burden

The vaginal lavage fluid was serially diluted and plated on a YPD agar plate containing ampicillin $(100 \mu \mathrm{g} / \mathrm{mL})$. The YPD agar plates were then incubated for $48 \mathrm{~h}$ at $30^{\circ} \mathrm{C}$, and the colonies were counted manually. The data was plotted as $\log \mathrm{CFU} / 100 \mu \mathrm{L}$ of vaginal fluid for each of the treatment groups.

(b) Histological analysis of the vaginal tissue

Following the lavage, the vaginal tract was excised longitudinally, washed twice with phosphate-buffered saline, and fixed in 10\% neutral-buffered formalin. The tissues were dehydrated with a gradual increase in alcohol concentrations followed by xylene. The tissues were then embedded in paraffin in an orientation that allowed cross-sectional cutting into $5 \mu \mathrm{m}$ thick sections. All the tissue sections were mounted on a poly-l-lysine-coated slide and stained with hematoxylin and eosin ( $\mathrm{H} \& \mathrm{E})$ as previously described [24]. The images were acquired using a Zeiss Axio Scope A1 microscope (Micro-Optics Precision Instruments, Fresh Meadows, NY, USA) with Zeiss Zen 2.3 software.

\subsection{Statistical Analysis}

The results reported here represent the geometric mean \pm standard error of the mean (SEM) from at least three representative experiments. The data were analyzed by one- 
way ANOVA followed by Dunnett's multiple comparison test using GraphPad Prism 8.0. Statistical significance was considered at $p<0.05$.

\section{Results and Discussion}

Antimicrobial drug discovery has been significantly impacted because of the large number of diverse chemical scaffolds naturally occurring in plants. The antibacterial and antifungal activity of CAR, a phenolic monoterpenoid, has been documented in various literature $[9,13,14]$, and CAR prodrugs are better candidates than CAR with respect to their physico-chemical properties (they are more water-soluble and less volatile) (Table 1). Additionally, the former possesses improved antimicrobial activity [14].

Table 1. Molecular properties of the CAR and the WSCPs.

\begin{tabular}{ccccc}
\hline Compounds & $\begin{array}{c}\text { Molecular } \\
\text { Weight (g/mol) }\end{array}$ & $\begin{array}{c}\text { Water } \\
\text { Solubility (mg/mL) }\end{array}$ & LogP $\boldsymbol{a}^{\boldsymbol{a}}$ & $\begin{array}{c}\text { Topological } \\
\text { Polar Surface } \\
\text { Area (TPSA) }\end{array}$ \\
\hline CAR & 150.22 & 0.11 & 2.82 & 20.23 \\
WSCP1 & 243.73 & 587 & 2.17 & 52.32 \\
WSCP2 & 257.76 & 191 & 2.53 & 52.32 \\
WSCP3 & 257.76 & 480 & 2.43 & 52.32 \\
\hline${ }^{a}$ Prediction SwissADME platforms. & & &
\end{tabular}

The prodrug approach of coupling with a suitable amino acid was used in the drug design of WSCP1-3 to increase the solubility. The WSCP1 was coupled with glycine, whereas the WSCP2 and the WSCP3 were coupled with L-alanine and $\beta$-alanine, respectively [14]. In the present study, CAR and the prodrugs were evaluated for the antimicrobial activity by the broth microdilution method. The MIC values of the WSCPs and the CAR against both $\mathrm{S} 1$ and $\mathrm{S} 2$ strains at $48 \mathrm{~h}$ exhibited the following trend in order of decreasing potency: WSCP1, WSCP2 > WSCP3 > CAR > FLU (Table 2).

Table 2. MIC values for the CAR and its prodrugs and the FLU in the S1 and the S2 strains of C. albicans ${ }^{1}$.

\begin{tabular}{ccc}
\hline \multirow{2}{*}{ Test Compounds } & \multicolumn{2}{c}{ Mean MIC $_{\mathbf{4 8 h}}$} \\
\cline { 2 - 3 } & S1 Strain & S2 Strain \\
\hline CAR & $8 \mathrm{mM}(1.2 \mathrm{mg} / \mathrm{mL})$ & $16 \mathrm{mM}(2.4 \mathrm{mg} / \mathrm{mL})$ \\
WSCP1 & $2 \mathrm{mM}(0.4 \mathrm{mg} / \mathrm{mL})$ & $2 \mathrm{mM}(0.4 \mathrm{mg} / \mathrm{mL})$ \\
WSCP2 & $2 \mathrm{mM}(0.5 \mathrm{mg} / \mathrm{mL})$ & $2 \mathrm{mM}(0.5 \mathrm{mg} / \mathrm{mL})$ \\
WSCP3 & $4 \mathrm{mM}$ & $4 \mathrm{mM}$ \\
FLU & $>16 \mathrm{mM}$ & $>16 \mathrm{mM}$ \\
\hline
\end{tabular}

${ }^{1}$ Unless otherwise indicated, all the MIC values represent the average of three experiments, each performed in triplicate.

In this study, WSCP1 was the most active prodrug against C. albicans, showing an MIC value equal to $2 \mathrm{mM}(0.4 \mathrm{mg} / \mathrm{mL})$ compared to that of FLU $(>16 \mathrm{mM})$, a drug of choice for the prevention and treatment of candidiasis.

The antifungal activity of CAR has been attributed to its effect on ergosterol biosynthesis, thereby disrupting the membrane integrity in a manner similar to FLU [13]. However, the present study was an attempt to assess whether CAR and its derivatives inhibit medium acidification by yeasts by reducing the activity of the plasma membrane H+-ATPase pump (Pma1p). The essential proton pump Pma1p, which maintains the intracellular $\mathrm{pH}$, is vital for yeast growth. In general, the intracellular $\mathrm{pH}$ in a yeast cell is maintained between 6.0 and 7.5 by Pma1p [25]. The fungal Pma1p extrudes protons from the inside of the yeast cell to the external environment by coupling ATP hydrolysis to ion transport, thereby making the extracellular medium acidic $[15,26]$. Mechanistic studies have shown the impact of other naturally occurring chemical compounds such as eugenol acting on the H+-ATPase 
pump $[25,27]$. Our study showed that CAR and WSCP1 inhibits proton pump extrusion by $\mathrm{S} 1$ and S2 strains with an $\mathrm{IC}_{50}$ concentration of approximately $2 \mathrm{mM}$ (Table 3), which was similar to the MIC concentration.

Table 3. Pma1p inhibition by the CAR and the WSCP1 in the S1 and the S2 strain of C. albicans.

\begin{tabular}{ccc}
\hline \multirow{2}{*}{ Test Compounds } & \multicolumn{2}{c}{ Mean IC $_{\text {50MA }}(\mathrm{mM})$} \\
\cline { 2 - 3 } & S1 Strain & S2 Strain \\
\hline CAR & $2.0 \pm 0.2$ & $1.9 \pm 0.6$ \\
WSCP1 & $1.6 \pm 0.7$ & $2.2 \pm 0.4$ \\
\hline
\end{tabular}

The effect of the test compounds on the acidification of the growth medium surrounding the S1 and S2 colonies was studied by incubating the cells with increasing concentrations $(0.1,0.3,1$, and $3 \mathrm{mM})$ of CAR and WSCP1 and assessing their inhibitory activity on acidification over a period of $30 \mathrm{~min}$ (Supplementary Figure S2). Further studies will help to shed more light on this proposed mechanism and other possible mechanisms of these compounds.

The data presented here with WSCP1-3, the hydrophilic derivatives of CAR with a slightly lower $\log \mathrm{P}$ than that of CAR [14], have shown an improvement in the antifungal activity against both the $S 1$ and the $S 2$ strains of $C$. albicans in vitro. Furthermore, considering the hydrophilic and hydrophobic balance of these compounds via the prodrug approach could help them dissolve in the microbial membrane and impair ergosterol biosynthesis, which is significant for the integrity of the fungal membrane.

Lastly, we have determined the potential efficacy and lower toxicity of CAR derivatives in lieu of frequently used antifungal drugs in a mouse model of VVC. VVC has always been a leading reason for women to visit a gynecologist at least once during their reproductive age. Owing to the rise in resistant strains, currently available antifungal treatments are unable to prevent recurrent VVC episodes, resulting in boundless discomfort which necessitates the investigation and identification of novel compounds with intriguing targets and antifungal efficacy.

For in vivo assessment, CAR and the most active prodrug, WSCP1, were formulated using various excipients such as Captex 300 and Kolliphor HS15 based on the previous preformulation studies with a view to achieve effective solubilization of hydrophobic drugs [28]. These excipients were previously used in a similar study by our lab as well as widely incorporated in a range of studies in the cosmetics and pharmaceutics domain. In vivo studies using these excipients did not exhibit reproductive health toxicity and skin irritation, thereby corroborating that these excipients are generally regarded as safe at the concentrations used here [29].

For implementing the mouse VVC model, estrogenizing the mouse is crucial since previous studies have shown a direct correlation of high estrogen levels with vaginal epithelium thickening and elevated glycogen content, resulting in increased susceptibility to VVC infection, which simulates the pregnancy or menstrual cycle conditions $[2,23,30]$. After the study period (day 6), mice were euthanized and vaginal lavage fluid was collected. The measurement of colony forming units (CFU / $100 \mu \mathrm{L}$ ) exhibited the maintenance of a robust vaginal fungal burden by day 6 (Figure 2). 


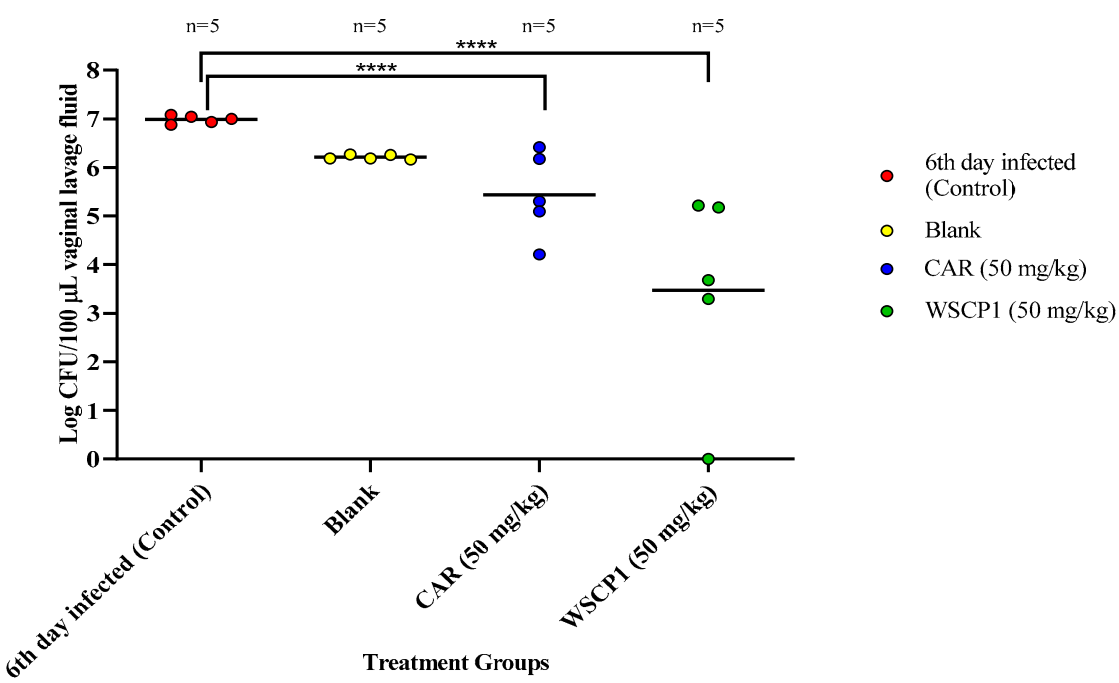

Figure 2. Effect of the CAR and the WSCP1 on VVC in a mouse model. ${ }^{* * * *} p<0.001$ relative to control.

The vaginal fungal burden indicated that the control group (infected, untreated) showed a high infection rate followed by blank, CAR (50 mg/kg), and WSCP1 (50 mg/kg). Treatment with the WSCP1 $(50 \mathrm{mg} / \mathrm{kg}$ ) formulation remarkably reduced the fungal burden by 120 -fold (Table 4). Mice treated with the CAR and the WSCP1 showed a high degree of variation in response to treatment, with one WSCP1-treated mouse showing a complete clearing of yeast. One could argue that this mouse represents an outlier point, but we do not believe it was an outlier. The mouse appeared healthy, and it was treated exactly like all the others in the cohort. There was no loss of vaginal lavage fluid or anything else that we observed that would lead to its exclusion from the study. While it remains unknown, the reason for the high variation in CAR- or WSCP1-treated mice is worthy of future study.

Table 4. Treatment groups with fold reduction compared to the control group.

\begin{tabular}{cc}
\hline Treatment Groups & Fold Reduction Compared to the Control \\
\hline 6th-day-infected (control) & 1.0 \\
Blank & 4.8 \\
CAR $(50 \mathrm{mg} / \mathrm{kg})$ & 8.8 \\
WSCP1 $(50 \mathrm{mg} / \mathrm{kg})$ & 122.2 \\
\hline
\end{tabular}

This was further substantiated by the characteristic parameters of inflammation and damage to the vaginal mucosa such as vaginal epithelial cell (VEC) hyperplasia, edema, and damage to the keratin layer. $\mathrm{H} \&$ E-stained vaginal tissues from the 6th-day group (infected, untreated) showed edema and hyperplasia of the VECs (Figure 3a), whereas the tissue sections from the blank-treated mice (Figure $3 b$ ) appeared healthy. Mice treated with CAR (50 mg/kg) (Figure 3c) and WSCP1 (50 mg/kg) (Figure 3d) showed slight epithelial distress (a loosely organized keratin layer) but otherwise appeared similar to blank-treated mice (Figure 3b). Further studies should be carried out to shed light on the formulation safety of CAR and WSCP1 to human vaginal epithelia. 

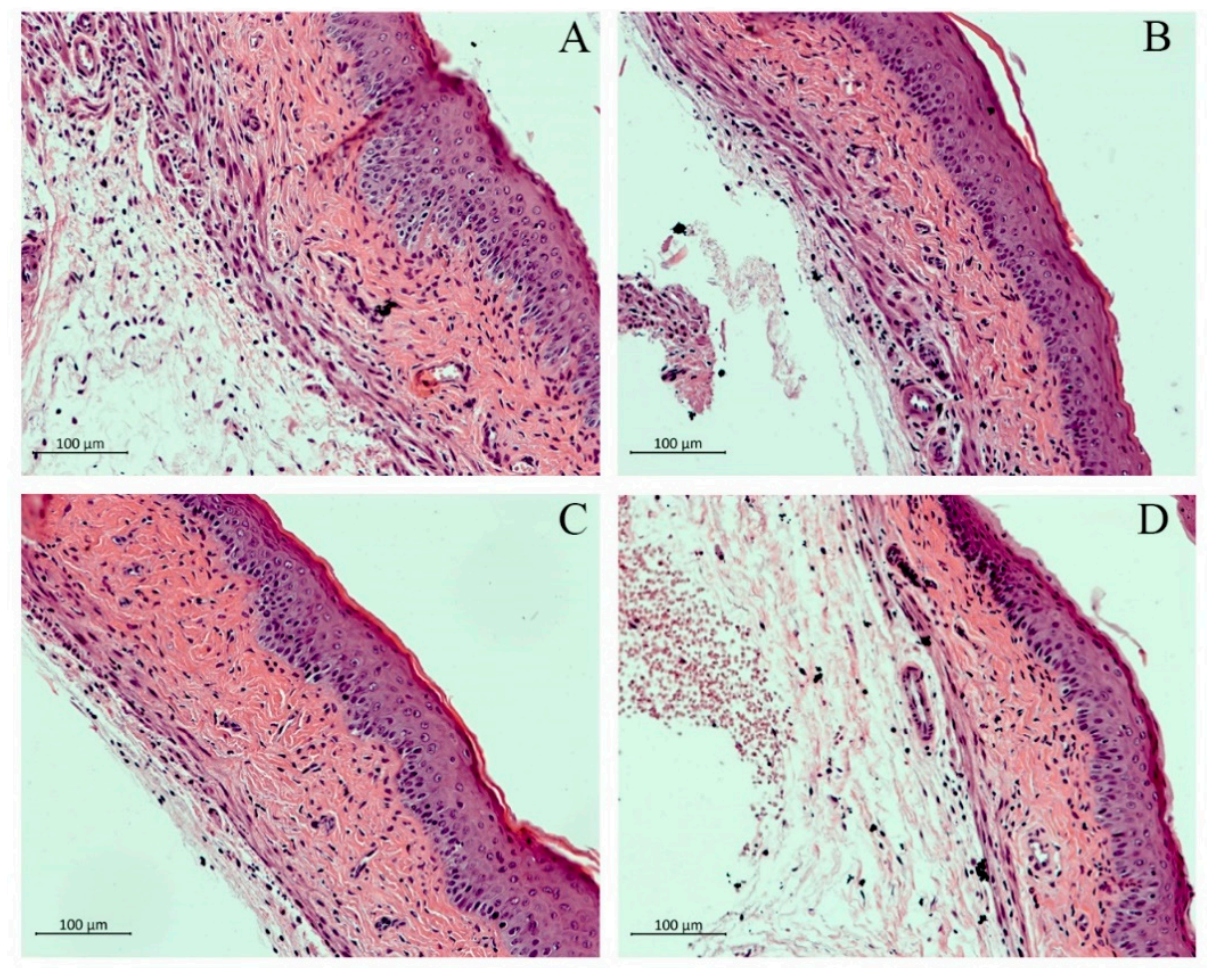

Figure 3. Histopathological analysis of vaginal tissues with $\mathrm{H} \& \mathrm{E}$ staining. Mouse vaginal tissue was excised longitudinally, fixed in $10 \%$ neutral-buffered formalin, and then embedded in paraffin. Each section of paraffin-embedded tissues was stained with $\mathrm{H} \& \mathrm{E}$ and then observed using alight microscope. (A) Naive group, (B) Blank, (C) CAR (50 mg/kg), and (D) WSCP1 (50 mg/kg). Magnification: $400 \times$; scale bars, $100 \mu \mathrm{m}$.

Essential oils are aromatic in nature and derived from plants. They exhibit a broad spectrum of activities, including antifungal properties. At least one report has found that the susceptibilities of FLU-resistant strains of C. albicans to oregano, thyme, and ginger essential oils were higher than those of the FLU-susceptible yeasts [31]. More recently, transmission and scanning electron microscopy analyses of $C$. albicans treated with oregano oil inhibited both the growth and the activity of the FLU-resistant yeast strain C. albicans ATCC 14053 more efficiently than clotrimazole [32]. Nazzaro and colleagues (2017) have extensively surveyed the literature and have summarized the antifungal effects of essential oils such as membrane disruption, cell wall inhibition, toxicity to mitochondria, or inhibition to drug efflux pumps [33]. The present study focused on CAR, an important component of oregano oil, and its water-soluble prodrug WSCP1 and found that these compounds inhibit the yeast plasma membrane H+-ATPase. Whether this is due to a direct effect of these drugs on the ATPase itself or an indirect effect due to mitochondrial toxicity or membrane disruption is an area of interest for future study in our lab. It will be interesting to also determine the extent to which oregano oil itself inhibits the Pma1p of $C$. albicans and reduces the fungal burden in the mouse model of VVC.

The rationale for selecting the test dose of $50 \mathrm{mg} / \mathrm{kg}$ for the CAR and the WSCP1 in the mouse experiment was based on three previously published in vivo reports. First, an acute toxicity study in mice found that the LD10 and LD50 of carvacrol were $546.8 \mathrm{mg} / \mathrm{kg}$ and $919 \mathrm{mg} / \mathrm{kg}$, respectively [34]. A second in vivo study in mice found that cognitive dysfunction associated with ethanol is alleviated by CAR at test doses of $50 \mathrm{mg} / \mathrm{kg}$ and $100 \mathrm{mg} / \mathrm{kg}$ [35]. Still yet another report found that CAR exhibited antinociceptive activity in mice at test doses of $50 \mathrm{mg} / \mathrm{kg}$ and $100 \mathrm{mg} / \mathrm{kg}$ [36]. Thus, we selected the $50 \mathrm{mg} / \mathrm{kg}$ test doses for the CAR and the WSCP1 used here based on these previous reports so that the test doses would fall well below the LD50 of CAR. Again, no such information is available 
for WSCP1 at the present time, but, since it is a CAR prodrug, we used the same test dose for both compounds.

The in vitro arm of the present manuscript also investigated the antifungal spectrum of CAR and its prodrugs by comparing the effects of these compounds in both an FLUresistant clinical isolate (S2) and a matched FLU-susceptible isolate from the same patient (S1) [18]. Indeed, the mechanism of FLU resistance in the S2 clinical isolate has been described previously [37] and is derived from a gain-of-function mutation in the transcription factor Upc2p, over-expression of the transporter Mdr1p, elevated expression of nine genes involved in ergosterol biosynthesis, and an increased expression of a mutated ERG11 gene coding for a mutated endogenous Erg11p, wherein G307S and G448E substitutions decrease the affinity of the enzyme for FLU [37,38]. Moreover, overexpression of Erg11p in the S2 strain leads to the increased production of lanosterol demethylase, which contributes to azole resistance of this disease-associated strain [37]. In the present study, we have discovered that CAR and its prodrugs are effective as antifungal agents, at least in part, via a mechanism that involves Pma1p inhibition, even when the yeast strains exhibit elevated Mdr1p and Erg11p. Hosseini and colleagues (2016) found that CAR exhibited antifungal activity in clinical isolates of FLU-resistant C. albicans [39]; however, the extent to which Pma1p was inhibited by CAR in said isolates was not investigated. Thus, the present study adds another aspect to the antifungal mechanism of action of CAR, which is Pma1p inhibition by CAR and its prodrugs.

The $\mathrm{IC}_{50 \mathrm{MA}}$ values measured here serve as an index of the Pma1p inhibitory activity of CAR and WSCP1. Whereas the WSCP1 exhibited similar MIC $(2 \mathrm{mM})$ and IC $\mathrm{I0MA}_{50}$ values $(2.2 \mathrm{mM})$, indicating a concordance between Pma1p inhibition and antifungal growth inhibition, this was not the case for the CAR. In the case of the CAR, the MIC values were four-fold higher in the S1 $(8 \mathrm{mM})$ and eight-fold higher in the S2 $(16 \mathrm{mM})$ when compared to the IC $50 \mathrm{MA}$ values for the CAR in said strains $(\sim 2 \mathrm{mM})$. This indicates that while Pma1p inhibition by CAR is observed to occur and may contribute to the antifungal activity, additional mechanisms of fungal toxicity may be utilized for CAR, including disrupted ergosterol biosynthesis, increased membrane permeability and fluidity, and disrupted cationic gradients across the cell membrane [13,40,41]. CAR has also recently found to alter the cholesterol content of the HIV-1 viral membrane, blocking HIV-1 entry into target cells [42]. The extent to which WSCP1 can carry out these types of effects remains to be investigated but is anticipated, at least to some extent, based on its prodrug status and structural similarity to CAR.

\section{Conclusions}

Exploring novel targets in fungi may provide new pharmacological candidates that can combat the resistant strains. Our study was undertaken to understand if CAR and its prodrugs work by blocking the H+-ATPase pump (Pma1p), a viable target since it is critical to fungi survival. We extended our work from in vitro to in vivo by utilizing a novel intravaginal formulation of CAR and WSCP1, thereby alleviating the problem of drugs with poor water solubility. Furthermore, we identified the potential efficacy of CAR and WSCP1 as a therapeutic intervention in the mouse model of VVC.

Supplementary Materials: The following are available online at https:/ / www.mdpi.com/article/ 10.3390/pharmaceutics13050633/s1. Figure S1: study design for intervention treatment of murine VVC; Figure S2: Medium acidification assay and inhibition of Pma1p by (a) CAR and (b) WSCP1.

Author Contributions: Conceptualization, B.B. and I.C.; methodology, S.M., X.L. and R.V.; software, L.M. and S.M.; validation, L.M., I.C., K.P. and B.B.; formal analysis, S.M., K.P. and B.B.; writingoriginal draft preparation, S.M. and B.B.; writing—review and editing, S.M., X.L., R.V., K.P., B.B., A.D.S., I.C. and L.M.; supervision, A.D.S.; funding acquisition, A.D.S. All authors have read and agreed to the published version of the manuscript.

Funding: This research was funded by the Italian Ministry of Education, University and Research (University “G. d'Annunzio” of Chieti-Pescara), grant number FAR 2019. 
Institutional Review Board Statement: The study received the approval of the Institutional Animal Care and Use Committee (IACUC) of St. John's University.

Informed Consent Statement: Not applicable.

Data Availability Statement: Data is contained within the article and supplementary material.

Acknowledgments: The authors thank the Department of Pharmaceutical Sciences for providing the funding for these experiments.

Conflicts of Interest: The authors declare no conflict of interest.

\section{References}

1. da Silva Dantas, A.; Lee, K.K.; Raziunaite, I.; Schaefer, K.; Wagener, J.; Yadav, B.; Gow, N.A.R. Cell biology of Candida albicans-host interactions. Curr. Opin. Microbiol. 2016, 34, 111-118. [CrossRef] [PubMed]

2. Cassone, A. Vulvovaginal Candida albicans infections: Pathogenesis, immunity and vaccine prospects. BJOG 2015, 122, 785-794. [CrossRef]

3. Bakkali, F.; Averbeck, S.; Averbeck, D.; Idaomar, M. Biological effects of essential oils-A review. Food Chem. Toxicol. 2008, 46, 446-475. [CrossRef] [PubMed]

4. Bouyahya, A.; Chamkhi, I.; Benali, T.; Guaouguaou, F.E.; Balahbib, A.; El Omari, N.; Taha, D.; Belmehdi, O.; Ghokhan, Z.; El Menyiy, N. Traditional use, phytochemistry, toxicology, and pharmacology of Origanum majorana L. J. Ethnopharmacol. 2021, 265, 113318. [CrossRef] [PubMed]

5. $\quad$ Le, N.T.; Donadu, M.G.; Ho, D.V.; Doan, T.Q.; Le, A.T.; Raal, A.; Usai, D.; Sanna, G.; Marchetti, M.; Usai, M.; et al. Biological activities of essential oil extracted from leaves of Atalantia sessiflora Guillauminin Vietnam. J. Infect. Dev. Ctries. 2020, 14, $1054-1064$. [CrossRef]

6. Swamy, M.K.; Akhtar, M.S.; Sinniah, U.R. Antimicrobial properties of plant essential oils against human pathogens and their mode of action: An updated review. Evid. Based Complement. Alternat. Med. 2016, 6, 1-21. [CrossRef]

7. Lambert, R.J.W.; Skandamis, P.N.; Coote, P.J.; Nychas, G.-J.E. A study of the minimum inhibitory concentration and mode of action of oregano essential oil, thymol and carvacrol. J. Appl. Microbiol. 2001, 91, 453-462. [CrossRef]

8. Bassolé, I.H.N.; Juliani, H.R. Essential Oils in Combination and Their Antimicrobial Properties. Molecules 2012, 17, 3989-4006. [CrossRef]

9. Cacciatore, I.; di Giulio, M.; Fornasari, E.; di Stefano, A.; Cerasa, L.S.; Marinelli, L.; Turkez, H.; di Campli, E.; di Bartolomeo, S.; Robuffo, I.; et al. Carvacrol codrugs: A new approach in the antimicrobial plan. PLoS ONE 2015, 10, e0120937.

10. Friedman, M. Chemistry and Multibeneficial Bioactivities of Carvacrol (4-Isopropyl-2-methylphenol), a Component of Essential Oils Produced by Aromatic Plants and Spices. J. Agric. Food Chem. 2014, 62, 7652-7670. [CrossRef]

11. Veldhuizen, E.J.A.; Tjeerdsma-van Bokhoven, J.L.M.; Zweijtzer, C.; Burt, S.A.; Haagsman, H.P. Structural Requirements for the Antimicrobial Activity of Carvacrol. J. Agric. Food Chem. 2006, 54, 1874-1879. [CrossRef] [PubMed]

12. Ultee, A.; Bennik, M.H.J.; Moezelaar, R. The Phenolic Hydroxyl Group of Carvacrol Is Essential for Action against the Food-Borne Pathogen Bacillus cereus. Appl. Environ. Microbiol. 2002, 68, 1561-1568. [CrossRef]

13. Ahmad, A.; Khan, A.; Akhtar, F.; Yousuf, S.; Xess, I.; Khan, L.A.; Manzoor, N. Fungicidal activity of thymol and carvacrol by disrupting ergosterol biosynthesis and membrane integrity against Candida. Eur. J. Clin. Microbiol. Infect. Dis. 2011, 30, 41-50. [CrossRef] [PubMed]

14. Marinelli, L.; Fornasari, E.; Eusepi, P.; Ciulla, M.; Genovese, S.; Epifano, F.; Fiorito, S.; Turkez, H.; Örtücü, S.; Mingoia, M.; et al. Carvacrol prodrugs as novel antimicrobial agents. Eur. J. Med. Chem. 2019, 178, 515-529. [CrossRef]

15. Perlin, D.S.; Seto-Young, D.; Monk, B.C. The plasma membrane H(+)-ATPase of fungi. A candidate drug target? Ann. N. Y. Acad. Sci. 1997, 834, 609-617. [CrossRef] [PubMed]

16. Yano, J.; Peters, B.M.; Noverr, M.C.; Fidel, P.L., Jr. Novel mechanism behind the immunopathogenesis of vulvovaginal candidiasis: "Neutrophil anergy". Infect. Immun. 2018, 86, e00684-17. [CrossRef] [PubMed]

17. Conti, H.R.; Huppler, A.R.; Whibley, N.; Gaffen, S.L. Animal Models for Candidiasis. Curr. Protoc. Immunol. 2014, 105, 19.6.1-19.6.17. [CrossRef]

18. Franz, R.; Ruhnke, M.; Morschhäuser, J. Molecular aspects of fluconazole resistance development in Candida albicans. Mycoses 1999, 42, 453-458. [CrossRef]

19. Koga-Ito, C.Y.; Lyon, J.P.; de Resende, M.A. Comparison between E-test and CLSI broth microdilution method for antifungal susceptibility testing of Candida albicans oral isolates. Rev. Inst. Med. Trop. South Paulo. 2008, 50, 7-10. [CrossRef]

20. Clinical and Laboratory Standards Institute. Reference Method for Broth Dilution Antifungal Susceptibility Testing of Yeasts. Approved Standard, 2nd ed.; NCCLS Document M27-A2; Clinicaland Laboratory Standards Institute: Wayne, PA, USA, 2002.

21. Perlin, D.S.; Brown, C.L.; Haber, J.E. Membrane potential defect in hygromycin B-resistant pma1 mutants of Saccharomyces cerevisiae. J. Biol. Chem. 1988, 263, 18118-18122. [CrossRef]

22. Orie, N.N.; Warren, A.R.; Basaric, J.; Lau-Cam, C.; Piętka-Ottlik, M.; Młochowski, J.; Billack, B. In vitro assessment of the growth and plasma membrane $\mathrm{H}+$-ATPase inhibitory activity of ebselen and structurally related selenium- and sulfur-containing compounds in Candida albicans. J. Biochem. Mol. Toxicol. 2017, 31, e21892. [CrossRef] 
23. Yano, J.; Fidel, P. Protocols for Vaginal Inoculation and Sample Collection in the Experimental Mouse Model of Candida vaginitis. J. Vis. Exp. 2011, 58, 3382. [CrossRef]

24. Tumu, H.C.R.; Cuffari, B.J.; Pino, M.A.; Palus, J.; Piętka-Ottlik, M.; Billack, B. Ebselen oxide attenuates mechlorethamine dermatotoxicity in the mouse ear vesicant model. Drug Chem. Toxicol. 2020, 43, 335-346. [CrossRef]

25. Bhatia, R.; Shreaz, S.; Khan, N.; Muralidhar, S.; Basir, S.F.; Manzoor, N.; Khan, L.A. Proton pumping ATPase mediated fungicidal activity of two essential oil components. J. Basic Microbiol. 2012, 52, 504-512. [CrossRef]

26. Serrano, R. Structure and function of proton translocating ATPase in plasma membranes of plants and fungi. Biochim. Biophys. Acta. 1988, 947, 1-28. [CrossRef]

27. Ahmad, A.; Khan, A.; Yousuf, S.; Khan, L.A.; Manzoor, N. Proton translocating ATPase mediated fungicidal activity of eugenol and thymol. Fitoterapia 2010, 81, 1157-1162. [CrossRef]

28. Vartak, R.; Menon, S.; Patki, M.; Billack, B.; Patel, K. Ebselen nanoemulgel for the treatment of topical fungal infection. Eur. J. Pharm. Sci. 2020, 148, 105323. [CrossRef] [PubMed]

29. Giusto, K.; Patki, M.; Koya, J.; Ashby, C.R.; Munnangi, S.; Patel, K.; Reznik, S.E. A vaginal nanoformulation of a SphK inhibitor attenuates lipopolysaccharide-induced preterm birth in mice. Nanomedicine 2019, 14, 2835-2851. [CrossRef] [PubMed]

30. Fidel, P.L.; Cutright, J.; Steele, C. Effects of Reproductive Hormones on Experimental Vaginal Candidiasis. Infect. Immun. 2000, 68, 651-657. [CrossRef]

31. Pozzatti, P.; Scheid, L.A.; Spader, T.B.; Atayde, M.L.; Santurio, J.M.; Alves, S.H. In vitro activity of essential oils extracted from plants used as spices against fluconazole-resistant and fluconazole-susceptible Candida spp. Can. J. Microbiol. 2008, 54, 950-956. [CrossRef] [PubMed]

32. Bona, E.; Cantamessa, S.; Pavan, M.; Novello, G.; Massa, N.; Rocchetti, A.; Berta, G.; Gamalero, E. Sensitivity of Candida albicans to essential oils: Are they an alternative to antifungal agents? J. Appl. Microbiol. 2016, 121, 1530-1545. [CrossRef]

33. Nazzaro, F.; Fratianni, F.; Coppola, R.; Feo, V. Essential oils and antifungal Activity. Pharmaceuticals 2017, 10, 86. [CrossRef]

34. Andre, W.P.; Ribeiro, W.L.; Cavalcante, G.S.; dos Santos, J.M.; Macedo, I.T.; de Paula, H.C.; de Freitas, R.M.; de Morais, S.M.; de Melo, J.V.; Bevilaqua, C.M. Comparative efficacy and toxic effects of carvacryl acetate and carvacrol on sheep gastrointestinal nematodes and mice. Vet. Parasitol. 2016, 218, 52-58. [CrossRef]

35. Wang, P.; Luo, Q.; Qiao, H.; Ding, H.; Cao, Y.; Yu, J.; Liu, R.; Zhang, Q.; Zhu, H.; Qu, L. The neuroprotective effects of carvacrol on ethanol-induced hippocampal neurons impairment via the antioxidative and antiapoptotic pathways. Oxid. Med. Cell Longev. 2017, 2017, 4079425. [CrossRef]

36. Cavalcante Melo, F.H.; Rios, E.R.; Rocha, N.F.; Citó Mdo, C.; Fernandes, M.L.; de Sousa, D.P.; de Vasconcelos, S.M.; de Sousa, F.C. Antinociceptive activity of carvacrol (5-isopropyl-2-methylphenol) in mice. J. Pharm. Pharmacol. 2012, 64, 1722-1729. [CrossRef]

37. Dunkel, N.; Liu, T.T.; Barker, K.S.; Homayouni, R.; Morschhäuser, J.; Rogers, P.D. A gain-of-function mutation in the transcription factor Upc2p causes upregulation of ergosterol biosynthesis genes and increased fluconazole resistance in a clinical Candida albicans isolate. Eukaryot. Cell. 2008, 7, 1180-1190. [CrossRef]

38. Perea, S.; Lopez-Ribot, J.L.; Kirkpatrick, W.R.; McAtee, R.K.; Santillan, R.A.; Martinez, M.; Calabrese, D.; Sanglard, D.; Patterson, T.F. Prevalence of molecular mechanisms of resistance to azole antifungal agents in Candida albicans strains displaying high-level fluconazole resistance isolated from human immunodeficiency virus-infected patients. Antimicrob. Agents Chemother. 2001, 45, 2676-2684. [CrossRef]

39. Hosseini, S.S.; Yadegari, M.H.; Rajabibazl, M.; Ghaemi, E.A. Inhibitory effects of carvacrol on the expression of secreted aspartyl proteinases 1-3 in fluconazole-resistant Candida albicans isolates. Iran. J. Microbiol. 2016, 8, 401-409.

40. Rao, A.; Zhang, Y.; Muend, S.; Rao, R. Mechanism of antifungal activity of terpenoid phenols resembles calcium stress and inhibition of the TOR pathway. Antimicrob. Agents Chemother. 2010, 54, 5062-5069. [CrossRef]

41. Xu, J.; Zhou, F.; Ji, B.P.; Pei, R.S.; Xu, N. The antibacterial mechanism of carvacrol and thymol against Escherichia coli. Lett. Appl. Microbiol. 2008, 47, 174-179. [CrossRef]

42. Mediouni, S.; Jablonski, J.A.; Tsuda, S.; Barsamian, A.; Kessing, C.; Richard, A.; Biswas, A.; Toledo, F.; Andrade, V.M.; Even, Y.; et al. Oregano Oil and Its Principal Component, Carvacrol, Inhibit HIV-1 Fusion into Target Cells. J. Virol. 2020, 94, e00147-20. [CrossRef] [PubMed] 